\title{
COMMENTARY
}

\section{Identifying patients at risk for augmented renal clearance in the ICU - limitations and challenges}

\author{
Mieke Carlier ${ }^{1,2}$ and Jan J De Waele ${ }^{* 1}$ \\ See related research by Udy et al., http://ccforum.com/content/17/1/R35
}

\begin{abstract}
Augmented renal clearance (ARC) is an important determinant of antibiotic exposure in critically ill patients, and identifying patients at risk is therefore an important goal. There is a growing body of evidence that a younger patient with a low to moderate degree of organ dysfunction typically is at risk of ARC and therefore decreased exposure to renally eliminated antibiotics. Mechanisms potentially involved, such as increased cardiac output, have, however, not been identified as appropriate surrogate markers, and the search for suitable alternatives to readily identify patients with ARC continues.
\end{abstract}

This commentary discusses the findings of Udy and colleagues about the augmented renal clearance in septic and traumatized patients [1]. Antibiotic therapy is a key element of the treatment of patients with severe sepsis and septic shock, and success of antibiotic therapy is determined by multiple factors. Susceptibility of the microorganism to the antibiotic is crucial, but reaching an adequate concentration of the drug is equally relevant.

In recent years several studies found that plasma concentrations of antibiotics - with beta-lactam antibiotics investigated most intensely - are highly variable in critically ill patients, with a considerable number of patients not reaching adequate concentrations. Several factors, such as increased volume of distribution, hypoalbuminemia and increased elimination from the circulation, have been found to be involved in this phenomenon. As most of these antibiotics are primarily cleared via the kidneys, renal elimination has been studied most extensively, and the state of increased

*Correspondence: jan.dewaele@UGent.be

'Department of Critical Care Medicine, Ghent University Hospital, Ghent, 9000 Belgium

Full list of author information is available at the end of the article elimination of drugs via the kidney has been coined 'augmented renal clearance' (ARC), which is defined as a creatinine clearance of $130 \mathrm{ml} /$ minute $/ 1.73 \mathrm{~m}^{2}$ or higher [2]. The incidence of ARC has mainly been studied in smaller studies, and depending on the cutoff used for its definition and on the studied population, the incidence varied from $30 \%$ to $85 \%$ [3-5].

In their article, Udy and colleagues confirm the high incidence of ARC in a trauma and sepsis population, and found that age, trauma as admission diagnosis and a Sequential Organ Failure Assessment (SOFA) score of 4 or less were independently associated with ARC. Although they provided us with new insights in the characteristics of patients at risk of ARC, they could not translate their results into easy to use clinical guidance.

Identifying patients who present with ARC is obviously a desirable goal, in order to appropriately treat patients with severe infections. However, evaluation of kidney function in the ICU is notoriously difficult, and conventional biomarkers such as serum creatinine or estimates of glomerular filtration rate, such as Cockcroft-Gault or Modification of Diet in Renal Disease (MDRD) formulas, are not reliable, which is also the case in patients with ARC [6]. At present, creatinine clearance can most conveniently be measured using 8 or $24 \mathrm{~h}$ calculated creatinine clearance. When these data are not available, the results of the current study can serve as a guide to screen for ARC.

In the study by Udy and colleagues, cardiac index correlated with creatinine clearance - albeit poorly - and was not retained in the multivariate analysis. Although the methodology for cardiac index measurement used could also have influenced this finding, it may also demonstrate that renal clearance is determined by multiple factors and the search for surrogate markers may be elusive.

Irrespective of the findings reported, several questions remain unanswered. The incidence of ARC in more diverse and other ICU populations has been incompletely explored. Also, the dynamics of ARC during ICU stay have not been studied. Better methods for the early recognition of patients with ARC are necessary - potentially 
novel biomarkers such as neutrophil gelatinase-associated lipocalin (nGAL; currently mainly investigated in the setting of acute kidney failure) are worth exploring. Finally, diagnostic accuracy of different methods to diagnose ARC, including measured creatinine clearance, needs urgently to be determined.

Other than focusing on factors associated with the determinants of changed pharmacokinetics of antibiotics, performing actual therapeutic drug monitoring (TDM) may be an alternative approach. This is currently standard for drugs that have a narrow therapeutic index such as aminoglycosides or glycopeptides, but for other - safer drugs, TDM may offer a solution to monitor efficacy rather than toxicity. However, TDM of beta-lactams is rarely used in routine practice because the potential benefits of TDM have not yet been established, and because of long turn-around time and high costs.

Abbreviations

ARC, augmented renal clearance; TDM, therapeutic drug monitoring.

\section{Acknowledgements}

$\mathrm{MC}$ is funded by the Research Foundation Flanders.

\section{Competing interests}

The authors declare that they have no competing interests.

\section{Author details}

'Department of Critical Care Medicine, Ghent University Hospital, Ghent, 9000 Belgium. ${ }^{2}$ Department of Clinical Chemistry, Immunology and Microbiology,

Ghent University Hospital, Ghent, 9000 Belgium.
Published: 16 April 2013

\section{References}

1. Udy A, Roberts J, Shorr A, Boots R, Lipman J: Augmented renal clearance in septic and traumatized patients with normal plasma creatinine concentrations: identifying at-risk patients. Crit Care 2013, 17:R35.

2. Udy AA, Varghese JM, Altukroni M, Briscoe S, McWhinney BC, Ungerer JP, Lipman J, Roberts JA: Subtherapeutic initial beta-lactam concentrations in select critically ill patients: association between augmented renal clearance and low trough drug concentrations. Chest 2012, 142:30-39.

3. Udy A, Boots R, Senthuran S, Stuart J, Deans R, Lassig-Smith M, Lipman J: Augmented creatinine clearance in traumatic brain injury. Anesthesia Analgesia 2010, 111:1505-1510.

4. Fuster-Lluch O, Geronimo-Pardo M, Peyro-Garcia R, Lizan-Garcia M: Glomerular hyperfiltration and albuminuria in critically ill patients. Anaesth Intensive Care 2008, 36:674-680.

5. Lautrette A, Phan TN, Ouchchane L, AitHssain A, Tixier V, Heng AE, Souweine $B$ : High creatinine clearance in critically ill patients with communityacquired acute infectious meningitis. BMC Nephrol 2012, 13:124.

6. Baptista JP, Udy AA, Sousa E, Pimentel J, Wang L, Roberts JA, Lipman J: A comparison of estimates of glomerular filtration in critically ill patients with augmented renal clearance. Crit Care 2011, 15:R139. doi:10.1186/cc12573

Cite this article as: Carlier M, De Waele JJ: Identifying patients at risk for augmented renal clearance in the ICU - limitations and challenges. Critical Care 2013, 17:130. 